\title{
Rapport de la Présidente
}

Gabriela Rüttimann

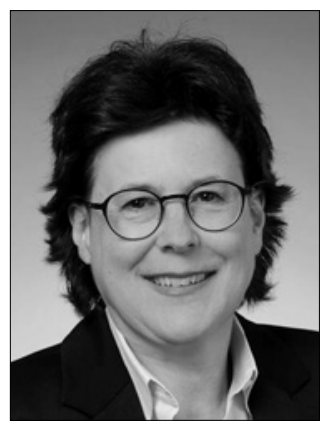

\section{L'ASP célèbre son 40ème anniversaire}

Le 3 mars 1979, l'Association Suisse des psychothérapeutes (SPV), aujourd'hui ASP, a été fondée à Bâle. À l'époque, cétait la première association à s'impliquer de manière constante dans la profession de psychothérapeutes en exercice en Suisse. Son but a été d'établir officiellement la psychothérapie, qui à l'époque nétait ni socialement reconnue ni légalement réglementée. L'Association s'est engagée dans une lutte pour la valorisation des normes de formation clairement définies et, exige un diplôme en psychologie ou une formation équivalente en sciences humaines comme condition préalable à la poursuite des études. La formation pluriannuelle doit être effectuée dans un institut de formation agréé.

Il a fallu près de 40 ans pour que la profession de psychothérapie soit légiférée par la PsyG (loi régissant les métiers de la psychologie). Depuis lors, la condition préalable à la formation psychothérapeutique est une étude psychologique; les études en sciences humaines ne sont plus approuvées. Notre vision de létude de la psychothérapie en tant que discipline scienti-

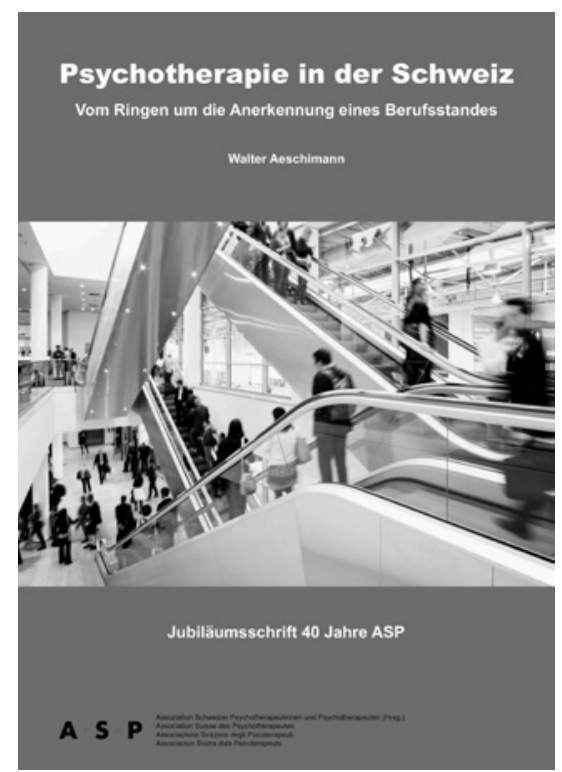

À l'occasion du 40ème anniversaire, I'ASP a publié un numéro intitulé Psychothérapie en Suisse - la lutte pour la reconnaissance d'une profession (Psychotherapie in der Schweiz - Vom Ringen um die Anerkennung eines Berufsstandes). fique indépendante reste inégalée - du moins en Suisse. l'Allemagne a cependant pris le pas et a adopté une loi correspondante à la fin de février. L'ASP a profité du 40ème anniversaire de l'association pour convier ses membres et ses invités au KOSMOS de Zurich à la célébration de cet anniversaire après l'assemblée générale ordinaire. La combinaison d'une salle de cinéma et d'une salle de club confortables, a offert une ambiance idéale à la fois pour la célébration, mais aussi pour l'agréable suite de la cérémonie qui a été accompagnée d'une gastronomie raffinée et de DJ Daniel, qui a interprété de la musique des années 70,80 et 90 .

Dans mon discours de cérémonie, j’ai résumé les 40 ans d'histoire, qui comprend des hauts et des bas, et qui sont décrits de manière frappante dans notre publication Psychothérapie en Suisse - La lutte pour la reconnaissance d'une profession (Psychotherapie in der Schweiz - Vom Ringen um die Anerkennung eines Berufsstandes) par l'historien et journaliste Walter Aeschimann. Il y est clairement précisé : L'histoire de l'Association est inextricablement liée au développement de la psychothérapie. La brochure aux récits vraiment passionnants, peut être obtenue auprès de notre secrétariat au prix de $35 \mathrm{CHF}$.

Un autre point culminant a été le dialogue entre Barbara Bleisch, philosophe et présentatrice de lémission télévisée "Sternstunde Philosophie", et Marcel Schär, professeur de psychologie et de psychothérapie à la Haute école zurichoise des sciences appliquées. Ils ont parlé du thème portant sur « le développement, l'authenticité, l'éthique - la recherche psycho-philosophique de traces ». La conversation a confirmé que la vision des personnes à partir d'un point de vue philosophique et psychologique fournit des informations extrêmement intéressantes dont elles peuvent bénéficier les unes des autres. Cela me ferait plaisir si nous pouvions continuer la discussion.

\section{La psychothérapie doit être incluse dans l'assurance de base}

Les associations ont mis une pression forte à Berne en demandant l'inclusion des traitements de psychothérapie dans l'assurance de base. Après la remise réussie de 3658 lettres de protestation adressées au conseiller fédéral Alain Berset, la campagne s'est poursuivie avec une pétition. Dans un délai de trois mois, 94422 signa- 
tures recueillies, ont été remises par les organisations participantes au Palais fédéral le 11 mars 2019. Cette fois-ci, non seulement les membres des associations de psychothérapie et de psychologie étaient impliqués, mais également ceux d'autres organisations. Laction a été menée dans la rue où toutes les personnes résidant en Suisse pouvaient signer.

\section{L'ASP en tant qu'organisation responsable}

Entre-temps, l'AAQ a recommandé l'accréditation de quatre des sept établissements de formation continue qui se sont inscrits au concept ASP Integral. Toutes les institutions attendent toujours la décision finale de l'EDI. Les recommandations sont fournies à des conditions qui doivent être soumises dans les deux ans suivant leur accréditation. En remplissant ces conditions, l'ASP, en tant qu'organisation responsable, devra jouer un rôle important, qui exige de la prudence et de la transparence lorsqu'il s'agit d'institutions de formation continue.

Étant donné que les points faibles se sont manifestés au cours du processus d'accréditation, l'ASP a l'intention d'en discuter avec les institutions de formation continue en vue du prochain cycle d'accréditation et d'en faire part aux autorités. Cet examen n'est pas seulement destiné aux établissements qui ont adhéré au concept ASP Integral, mais c'est également pour inviter les instituts, qui sont réunis dans la Conférence de la Charte, afin de rassembler le plus d'expérience possible.

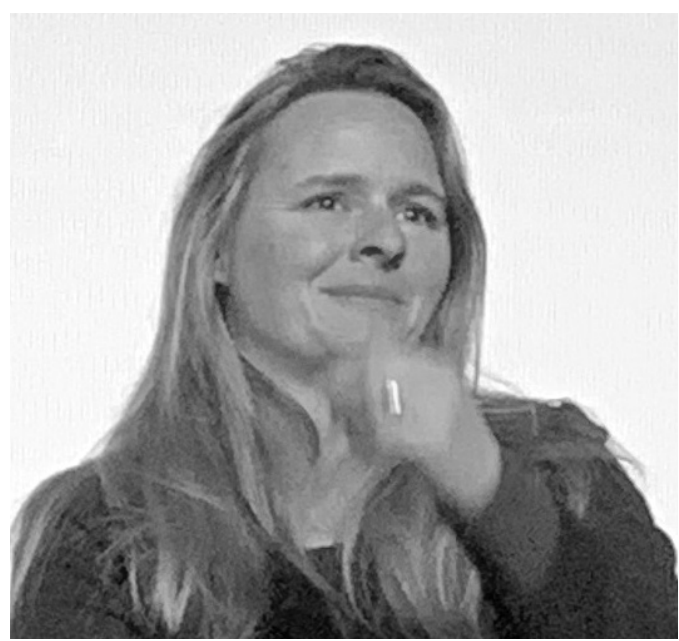

Le nouvelle membre du Conseil d'administration Sandra Feroleto représente I'ASP en Suisse romande.

\section{Formation continue : \\ Un must pour les psychothérapeutes}

Même après l'introduction du PsyG, ni l'Office fédéral de la santé ni les cantons ne semblent intéressés à savoir si les psychothérapeutes participent ou non à des cours de formation continue dans le sens de garantir la qualité de leur exercice professionnel. Le fait de suivre régulièrement des cours de formation n'a pas d'incidence sur l'attribution de licences de formation professionnelle, ni sur l'entrée dans le PsyReg. Les associations professionnelles considèrent donc qu'il est de leur devoir de s'occuper de cette question. Il est

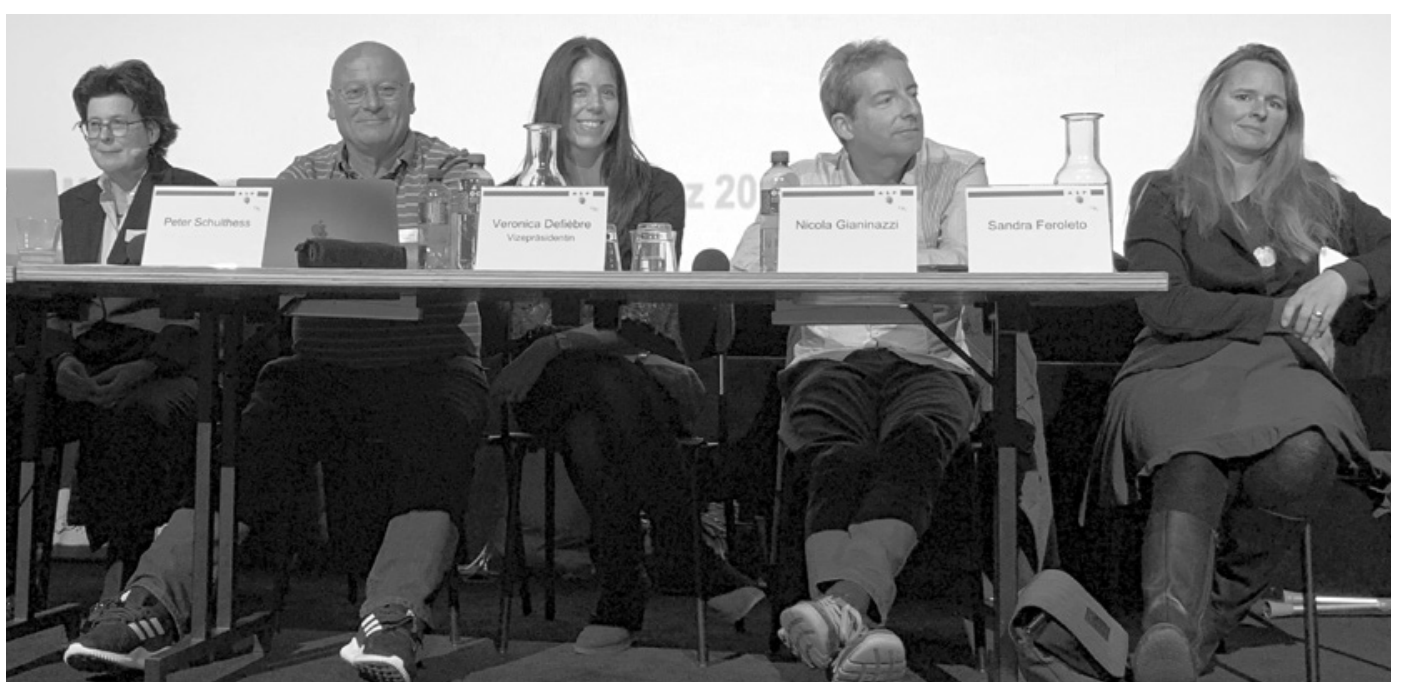

Le Conseil d'administration de l'ASP est à nouveau complet (de gauche à droite) : Gabriela Rüttimann (présidente), Peter Schulthess, Veronica Defièbre (vice-présidente), Nicola Gianinazzi, Sandra Ferolato 


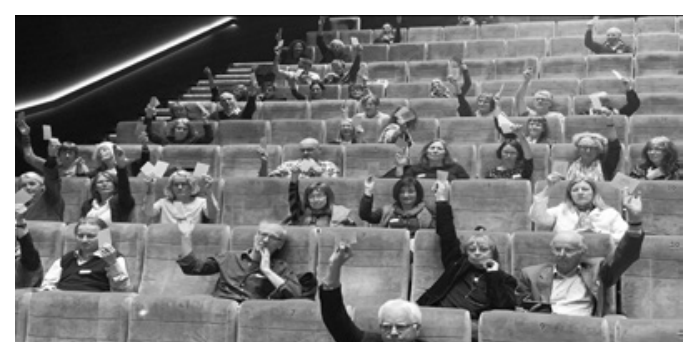

À l'Assemblée générale, pratiquement toutes les propositions ont été approuvées à l'unanimité.

logique qu'entre les associations, il y ait une certaine harmonisation des preuves de formation fournie, même si la mise en cuvre peut être différente. Il est important pour nous de ne pas rendre la vie inutilement difficile aux membres et délaborer une incitation qui leur crée une valeur ajoutée. Un règlement correspondant est en cours de préparation et sera publié dans nos canaux d'information une fois qu'il a été complété.

\section{Évaluation d'expertises de psychothérapeutes dans des procès}

Après avoir reçu diverses demandes dévaluation de psychothérapeutes dans des affaires judiciaires et par le fait que cependant rien n'ait été annoncé à ce sujet, nous nous sommes informés directement auprès du tribunal d'instance de Zurich.

Le traitement des rapports d'experts n'est soumis à aucune obligation de la part du tribunal. Selon leurs informations, cependant, tous les patients ont le droit de commander un rapport psychothérapeutique privé et de le joindre aux documents du tribunal comme preuve. Cela signifie que les avis des psychothérapeutes sont généralement approuvés. Il revient toutefois au juge, de par sa discrétion, de décider de la manière dont il reconnaît cela. Le même traitement s'applique par ailleurs aux rapports commandés par les autorités, ainsi qu’aux rapports de médecins traitants. La question de savoir si les tribunaux d'autres cantons traitent lévaluation des avis d'experts de la même manière devrait être examinée au cas par cas.

\section{Renforcement du rôle d'une association professionnelle}

Entre-temps, tous les psychothérapeutes en Suisse sont probablement inscrits au registre de la profession de psychologie. Cette entrée est assimilée à la reconnaissance fédérale, ce qui renforce sans aucun doute la profession. C'est dans lélaboration des directives juridiques et éthiques que les associations professionnelles occupent toujours une fonction importante, mais cela fait cependant entièrement défaut. En outre, les médiateurs et les organes de plaintes doivent être maintenus à la fois pour le patient et pour les membres, une tâche que nous menons depuis des années. L'ASP sert également de centre de traitement des plaintes de psychothérapeutes dans la formation de ces instituts qui sont affiliés à la Conférence de la Charte.

\section{Gabriela Rüttimann est présidente de l'ASP.}

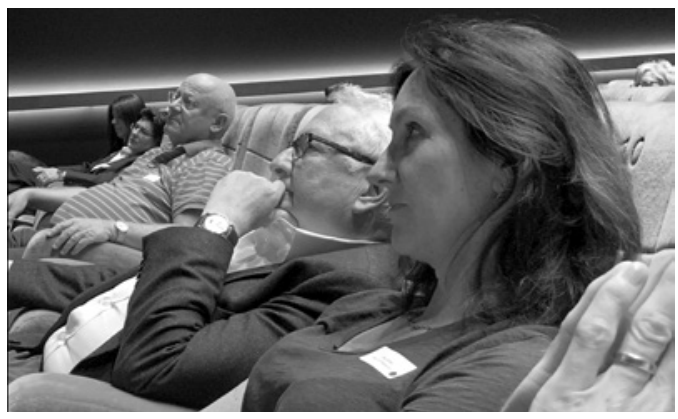

Une partie des leaders de l'ASP et du FSP dans la coexistence pacifique (de gauche à droite) : Veronica Defièbre, Gabriela Rüttimann, Peter Schulthess, Samuel Rom, Yvik Adler

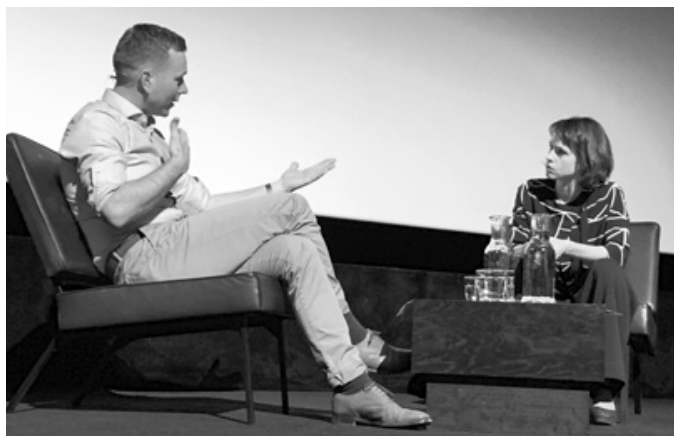

Le philosophe Dr. Barbara Bleisch en échange avec le Prof. Dr. Marcel Schär sur le thème " développement, authenticité, éthique - une recherche psycho-philosophique de traces » 